\title{
Review of Common Occupational Hazards and Safety Concerns for Nuclear Medicine Technologists*
}

\author{
Norman E. Bolus \\ Nuclear Medicine Technology Program, Department of Diagnostic and Therapeutic Sciences, School of Health Professions, University of \\ Alabama at Birmingham, Birmingham, Alabama
}

The purpose of this article is to address common occupational hazards and safety concerns of nuclear medicine technologists. There are many possible occupational hazards, but this review is intended to concentrate on common hazards and safety concerns. These include radiation safety issues and concerns about the possibility of developing latent diseases, such as eye cataracts or cancer; pregnant workers and radiation safety issues; biohazard concerns associated with patient body fluids; possible low-back pain from moving heavy equipment and performing patient transfers; and possible repetitive trauma disorders, such as carpal tunnel syndrome, from computer work. Suggestions are made regarding how to identify potential hazards and avoid them. After reading this article, nuclear medicine technologists should be able to explain the importance of the as-low-asreasonably-achievable concept, discuss the possible effects of ionizing radiation on the adult and the developing fetus, list several basic principles to avoid injury to the back, list and describe the more common repetitive trauma disorders or injuries and how to avoid them, and list and describe the biohazard safety issues that nuclear medicine technologists face and how to develop policy to minimize exposure risk.

Key Words: radiation safety; occupational hazards; biohazard safety; patient transfer techniques; cumulative trauma disorders

J Nucl Med Technol 2008; 36:11-17

DOI: 10.2967/jnmt.107.043869

$\mathbf{R}$ adiation safety is the main health issue of concern to a nuclear medicine technologist (NMT). Ionizing radiation is a known carcinogen at high doses, and clinical symptoms are known to be associated with chronic low-dose exposure. These include possible direct chromosomal changes, indirect

\footnotetext{
Received May 29, 2007; revision accepted Aug. 15, 2007.

For correspondence or reprints contact: Norman E. Bolus, MPH, CNMT, Room 444, Richard M. Scrushy Bldg., 1705 University Blvd., Birmingham, AL 35294-1212.

E-mail: Bolusn@uab.edu

*NOTE: FOR CE CREDIT, YOU CAN ACCESS THIS ACTIVITY THROUGH THE SNM WEB SITE (http://www.snm.org/ce_online) THROUGH MARCH 2010. Those without Internet access can obtain a hard copy of the test by calling 703-708-9000, ext. 1247.

COPYRIGHT @ 2008 by the Society of Nuclear Medicine, Inc.
}

free-radical production, and cataract formation. The lens of the eye contains radiosensitive cells that may be damaged or even destroyed by ionizing radiation exposure. Because the body is not able to naturally remove these damaged cells, they may accumulate to the point of causing cataract formation in the lens of the eye. The formation of cataracts caused by ionizing radiation is termed radiation cataractogenesis. The extent of opacity and chance of occurrence are proportional to the ionizing radiation dose and follow a threshold, nonlinear dose-response relationship. The threshold dose is approximately $2 \mathrm{~Gy}$ (200 rads). At doses greater than 7 Gy (700 rads), everyone irradiated would develop cataracts. The average latency period is thought to be approximately 15 y. High-linear-energy-transfer ionizing radiation has a high relative biological effectiveness for cataractogenesis, by a factor of 2 or more. The duration of exposure and the total dose seem to affect cataract formation. The shorter the duration and the higher the dose (above 2 Gy), the faster cataract formation occurs. In other words, chronic ionizing radiation exposure at low doses seems to decrease the efficiency of cataractogenesis (1).

In most cases, for low-dose radiation exposure, repair of damaged cells is likely, with no adverse effects occurring. The question remains as to whether chronic low-dose ionizing radiation actually leads to latent adverse effects, such as cancer induction, or not. From the scientific literature and data concerning low-dose exposure, there appears to be a threshold level, of 0.05-0.1 Sv (50-100 rems). However, regulatory agencies, such as the Nuclear Regulatory Commission, err on the side of caution and have adopted the no-threshold, linear dose-response curve and the as-lowas-reasonably-achievable (ALARA) concept. The basics of radiation safety should be taught early in an NMT career. They include the traditional 3 tenets of radiation safety: time, distance, and appropriate shielding (2). Estimated radiation exposure for NMTs ranges from 0.2 to $2 \mu \mathrm{Sv} / \mathrm{h}$, depending on whether NMTs are scanning patients (lower dose) or performing injections on patients (higher dose). Although these numbers are relatively low, it is best to adhere to the ALARA principle at all times (3). The following is a brief review of the 3 tenets of radiation safety. 


\section{RADIATION SAFETY ISSUES}

\section{Time}

It is intuitive to realize that the more time spent around a radioactive source, the more exposure or dose an individual will receive. Dose and dose rate are 2 different things. The dose is the total amount of ionizing radiation received over a time period. For instance, a monthly or quarterly dosimetry report shows dose received. The dose rate is the amount of ionizing radiation that an individual will receive if he or she stays in a certain location for a certain amount of time. For example, a dose rate of $0.3 \mathrm{mSv} / \mathrm{h}(30 \mathrm{mrem} / \mathrm{h})$ means that in order for an individual to receive a total dose of $0.15 \mathrm{mSv} / \mathrm{h}$ (15 mrem), he or she will need to stay in that one site for $30 \mathrm{~min}$ without moving. This fact should be self-evident; however, complacency in the workplace can lead to incidents that might increase an individual's ionizing radiation dose or exposure through poor work habits. For example, a technologist may be in the habit of answering a telephone or taking breaks in areas around a radioactive storage site. Doing so could lead to increased exposure over time. People should be vigilant in the workplace and periodically monitor, with a geiger counter or other survey instrument, possible sites where they could receive radiation exposure. It may be possible for people to modify their behaviors and change where they take breaks or answer a telephone to very simply reduce overall radiation exposure. These are simple solutions that anyone can use to reduce radiation exposure.

\section{Distance}

The second tenet of radiation safety is distance from a radioactive source. The greater the distance, the less the radiation received, by a factor of 1 over distance squared; this concept is more commonly referred to as the inverse square law. This fundamental principle of radiation exposure involves the distance that ionizing radiation must travel from a point source to an individual. This concept can be likened to a fireplace giving off heat. As an individual backs away from the fireplace, the warmth from it decreases by the inverse square law. The same is true of radiation exposure. Using the equation $\mathrm{I}_{1}\left(\mathrm{D}_{1}\right)^{2}=\mathrm{I}_{2}\left(\mathrm{D}_{2}\right)^{2}$, where $\mathrm{I}$ is intensity of source and $\mathrm{D}$ is distance from source, one can determine either the exposure rate or the distance needed to attain a certain exposure rate. If 3 parts of the equation are given, the fourth part can be determined by use of simple algebra. This type of exposure control method is commonly referred to as the least expensive way to control radiation exposure. Once again, it would be prudent to occasionally conduct a direct survey of the work area with a survey meter to assess whether distance can be increased to reduce radiation exposure.

\section{Appropriate Shielding}

The third tenet of radiation safety is greatly dependent on the type of ionizing radiation being used. Is the concern $\alpha$-radiation, $\beta$-radiation, $\gamma$-radiation, positron radiation, $x$-ray radiation, or a combination of any of these? The answer to that question is very important in determining the appropriate shielding to use and what the major health concern is.

$\alpha$-Radiation is, generally, not shielded. Its penetrating ability is so poor that it cannot pass through a piece of tissue paper or the thickness of human skin. The main health concern with an $\alpha$-source is the possibility of internal contamination, which would place the source close to radiosensitive organs of the body, such as bone marrow or the thyroid. Although $\alpha$-sources have low penetrating ability, they have high linear energy transfer, which results in a great deal of potential cellular damage from $\alpha$-radiation when it is internalized. There are 3 main ways to internalize a radiation source: absorption, ingestion, or inhalation. Basic laboratory guidelines, such as not eating, drinking, smoking, or applying cosmetics in a laboratory or clinic, are designed to minimize possible internal contamination with any xenobiotic agent (of which radiation is one) by ingestion. Wearing gloves and laboratory coats or other personal protective equipment (PPE) is designed to minimize or eliminate absorption of radiation through intact skin. Cuts and abrasions can increase the likelihood of absorption, and an individual should never try to decontaminate radioactive contamination of the skin by using abrasive brushes or pads or caustic chemicals, such as bleach. Doing so can increase the possibility of contamination via the absorption route. The best method for decontaminating skin is to wash with a mild detergent or dishwashing soap. For more severe contamination on the hands, the suggested method is to wear a glove for $24 \mathrm{~h}$ and sweat the radioactive contamination out. Good laboratory practices should prevent internalizing $\alpha$-radiation.

There are 2 concerns regarding the appropriate shielding of $\beta$-radiation. First, $\beta$-radiation is more penetrating than $\alpha$-radiation and travels in zig-zag or contorted paths. $\beta$-Radiation requires heavier types of shielding than $\alpha$-radiation, but an individual must be cautious about using high-z (atomic)number materials, such as lead, when $\beta$-radiation is present. This is the second concern, because high-z-number materials can cause bremsstrahlung radiation to form. Bremsstrahlung radiation consists of characteristic $\mathrm{X}$-rays that occur when the free electron of $\beta$-radiation knocks out an inner shell electron of high-z-number materials. Outer electrons fall in to replace the inner shell electron loss and give off x-ray energy that is dependent on the level in which the electron was located. This effect is not seen with low-znumber materials, such as Plexiglas, glass, and plastic. These types of shielding materials are used for $\beta$-radiation because of the bremsstrahlung radiation effect. If an individual is using an isotope, such as ${ }^{131} \mathrm{I}$, that has a mixed radiation emission of both $\gamma$-radiation and $\beta$-radiation, then he or she must shield the $\beta$-radiation first and the $\gamma$-component second because high-Z-number materials, such as lead, are needed to effectively shield $\gamma$-radiation. A plastic-coated lead shield is used for ${ }^{131} \mathrm{I}$ because the plastic will shield the $\beta$-radiation, and a sufficient thickness of lead will shield the $\gamma$-emission.

$\gamma$-Radiation, positron radiation, and $\mathrm{x}$-ray radiation should be shielded with high-z-number materials as stated 
earlier. The amount of shielding material is determined by the half-value layer (HVL) of the absorber and the energy of the $\gamma$-radiation, positron radiation $(511 \mathrm{keV})$, or $\mathrm{x}$-ray radiation. The HVL reduces the amount of the incident radiation to one half its original value. In general, unless extremely high levels of radiation are being used, $10 \mathrm{HVLs}$ are needed to reduce the incident radiation to the background radiation amount. Table 1 shows the HVLs for many isotopes commonly used in nuclear medicine. There are some differences between references as to the exact HVLs of some absorbers, primarily related to the purity of the HVL in question. As the energy of the radioisotope increases, the required thickness of the absorber increases. Also, as the density of the absorber increases, the amount needed for $1 \mathrm{HVL}$ decreases (6).

\section{Pregnant Workers}

To become declared as a pregnant worker in nuclear medicine, a woman must voluntarily, in writing, notify a supervisor. Otherwise, the law states that no matter how obvious a pregnancy may be, it cannot be acknowledged by coworkers or supervisors, and no special precautions will be instituted by a facility. Nondisclosure should be considered very carefully and is not recommended, for several obvious reasons. The first trimester, or organogenesis stage of embryonic development, is the most susceptible to radiation exposure because a great deal of cell division and stem cell development are occurring. These types of cells are very radiosensitive. Therefore, it is recommended that the following safety precautions be taken once a declaration of pregnancy is made. The woman should wear a wraparound lead apron that has a sufficient thickness of lead to attenuate the radioisotopes used at the clinical site to background levels of radiation exposure. For ${ }^{99 \mathrm{~m}} \mathrm{Tc}$, this thickness of lead would be at least $0.25 \mathrm{~cm}$. ALARA amounts of radiation exposure should be applied, as should the tenets of time and distance. A woman should be excused from performing radiotherapy procedures, such as ${ }^{131} \mathrm{I}$ therapy, for the duration of the pregnancy.

Positron emission studies present a special problem because most lead aprons are not sufficiently thick to

TABLE 1

HVLs for many isotopes commonly used in nuclear medicine

\begin{tabular}{lcc}
\hline Radioisotope & Main energy $(\mathrm{keV})$ & $\mathrm{HVL}$ of $\mathrm{Pb}(\mathrm{cm})$ \\
\hline${ }^{99 \mathrm{~m} T \mathrm{Tc}}$ & 140 & $\sim 0.02$ \\
${ }^{131} \mathrm{I}$ & 364 & $\sim 0.30$ \\
${ }^{133 \mathrm{Xe}}$ & 81 & $\sim 0.03$ \\
${ }^{111} \mathrm{In}$ & 245 & $\sim 0.10$ \\
${ }^{137} \mathrm{Cs}$ & 662 & $\sim 0.65$ \\
${ }^{201} \mathrm{TI}$ & 70 & $\sim 0.03$ \\
${ }^{125} \mathrm{I}$ & 35.5 & $\sim 0.01$ \\
\hline
\end{tabular}

Data were obtained from online sources $(4,5)$. effectively attenuate the 511-keV photons to background radiation levels. Because radiation is most harmful at the point at which it stops, if a woman does not use a sufficient thickness of an absorber to reduce emissions to background levels, then more harm may be created by slowing the radiation to the point at which it stops in the body. The bestcase situation would be to postpone exposure to positron emissions until at least after the first trimester, or organogenesis stage, of the pregnancy.

Once a declaration of pregnancy is made, a fetal dose is determined by the inclusion of a fetal whole-body dosimeter, which is worn at waist level under a lead apron, if one is used. Fetal exposure should not exceed $500 \mathrm{cSv}$ (mrem) spread over the duration of the 9-mo gestation period. If this 500-cSv level has already been exceeded before the declaration of the pregnancy, then the amount is reduced to an additional 50 $\mathrm{cSv}$ over the remaining gestation period. As Table 2 shows, the susceptibility of the fetus to radiation exposure decreases as the pregnancy approaches full term (7).

\section{Possible Fetal Radiation Exposure Effects}

Some possible side effects of fetal radiation exposure (other than cancer) include growth retardation, reduction in IQ, mental retardation, and possible major malformations (Table 2) (8). All of these are dependent on the dose and when the exposure of the fetus occurs after fertilization. For amounts below 0.05 Gy (5 rads), noncancer health effects are not detectable. At $0.05-0.50$ Gy (5-50 rads), up to $2 \mathrm{wk}$ after fertilization, the incidence of failure to implant may increase slightly, but surviving embryos will probably have no significant (noncancer) health effects. This is the "all-ornone effect"; either the embryo dies at this stage or survives with no significant health effects. During the organogenesis stage, at 2-7 wk after fertilization, with an exposure of $0.05-0.50$ Gy (5-50 rads), the embryo may have a slight increase in the incidence of major malformations, and growth retardation is possible. This is the most sensitive stage for malformation appearance in the embryonic growth cycle because a great deal of cell division is occurring. At 8-15 wk, the fetogenesis stage, with exposure to $0.05-0.50 \mathrm{~Gy}$ (5-50 rads), growth retardation of the fetus is possible. There can be a reduction in IQ of up to 15 points, depending on the dose, and severe mental retardation of up to $20 \%$ can occur, depending on the dose. At 16 wk or more after fertilization, with exposure to $0.05-0.50$ Gy (5-50 rads), no noncancer health effects are likely to occur. At greater than 0.50 Gy (50 rads), the expectant mother may experience acute radiation syndrome, depending on her whole-body dose. At up to 2 wk after fertilization, with exposure to greater than 0.5 Gy (50 rads), the incidence of failure to implant will likely be large, with 1 Gy (100 rads) killing 50\% of the embryos and 5 Gy (500 rads) killing $100 \%$ of the embryos. If an embryo does survive, then it will probably have no significant (noncancer) health effects. During the organogenesis stage, at 2-7 wk after fertilization, with exposure to 0.50 Gy or 


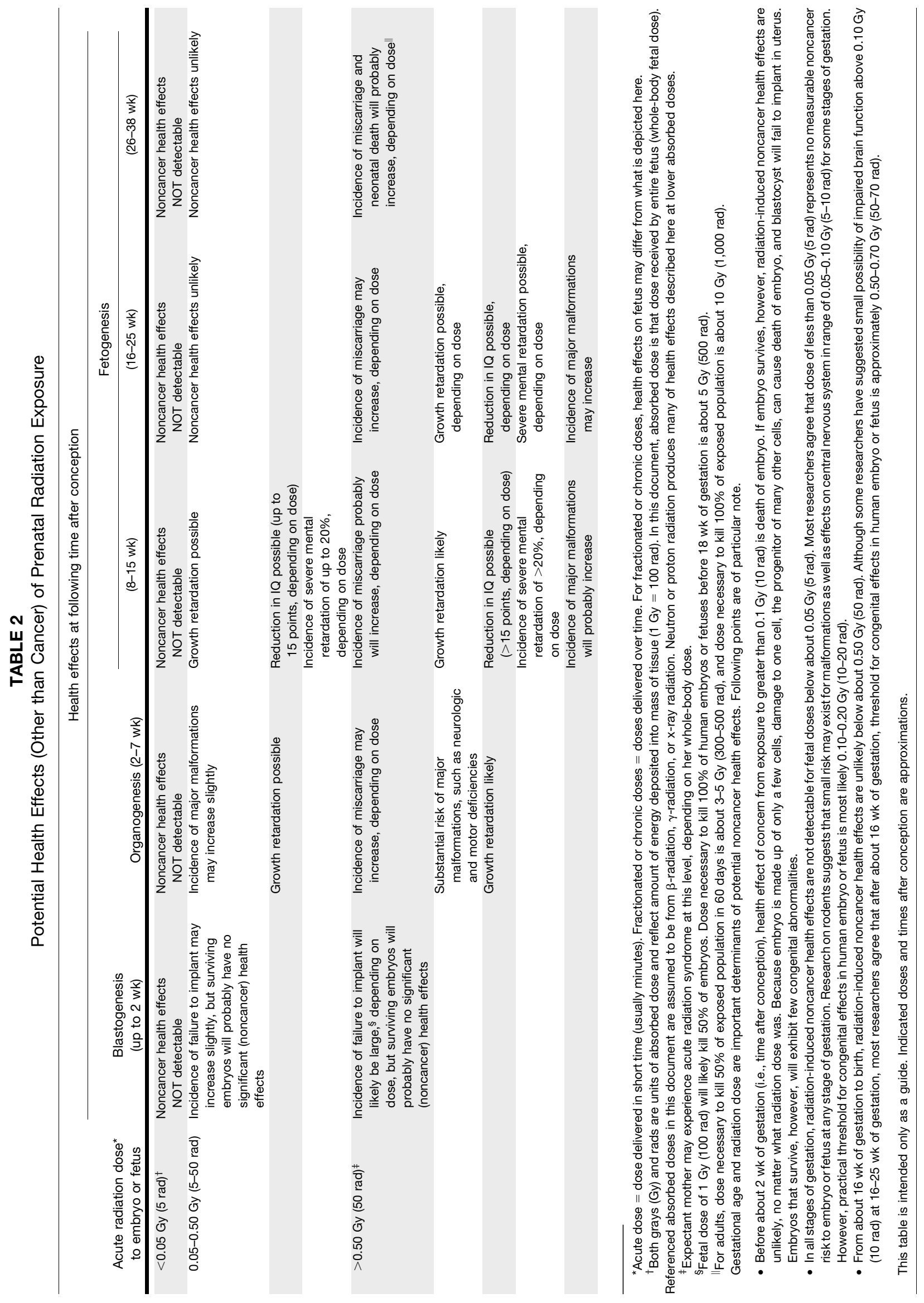

14 Journal of Nuclear Medicine Technology • Vol. 36 • No. 1 • March 2008 
greater, the incidence of miscarriage may increase, depending on the dose; there is a substantial risk of major malformations, such as neurologic and motor deficiencies; and growth retardation is likely. At $8-15 \mathrm{wk}$ after fertilization, at a dose of greater than $0.50 \mathrm{~Gy}$ ( $50 \mathrm{rads}$ ), in addition to what is seen at the previous interval, there can be a reduction in IQ of greater than 15 points and an incidence of severe mental retardation of greater than $20 \%$, depending on the dose. The major effect after $16 \mathrm{wk}$ of gestation is an increase in miscarriage, depending on the dose.

\section{Biohazard Safety Issues}

There are over 200 different biologic agents known to produce infections and allergenic, toxic, or carcinogenic reactions in workers. These agents and illnesses include microorganisms, such as viruses, bacteria, and fungi, capable of producing infection and allergies; allergens and toxins generated by plants, which can produce dermatitis, rhinitis, or asthma; protein allergens from vertebrate animals, such as dander, hair, and saliva; arthropod-induced illnesses, such as allergic reactions or inflammation caused by a bite or sting from an insect; and parasite-induced illnesses, such as schistosomiasis, caused by Schistosoma, or roundworm infestations, caused by Ascaris.

Many factors actually contribute to the possibility of exposure and then infection with a disease-causing agent. These include modes of transmission, route of entry, infectious dose, viability and virulence of the agent, and host susceptibility. Modes of transmission that would be associated with clinical nuclear medicine work include contact and vector-borne and airborne transmissions. Under contact transmission, there are direct and indirect methods, such as direct contact with an infected individual, infectious material spilled or splashed onto mucous membranes, irritated skin, or open cuts. The main defenses against direct contact are standard precautions (formally known as universal precautions), which include PPE, such as gloves, long-sleeved knee-length laboratory coats, or scrubs. Indirect contact includes transmission via some fomite; that is, some sort of inanimate object (e.g., countertop, bed, or camera) becomes contaminated, and the infectious agent is then transmitted to a host. Vector-borne transmission would be less likely in a clinical setting but would be a concern when a disease could develop through a living vector (e.g., mosquito, flea, or tick) biologically or mechanically transmitting a causative agent through a bite directly on the skin. Airborne transmission may occur through the inhalation of infectious airborne particles. Workers may be exposed by contact after the deposition of droplets, through splashing or spilling, onto surfaces, equipment, and personnel. Routes of entry for any infectious disease-causing agent include inhalation, ingestion, penetration through irritated skin, and contact with mucous membranes of the nose, eyes, and mouth. Ingestion may occur through hand-to-mouth contamination, such as eating, drinking, or smoking in the work area. Contaminated objects, such as hypodermic needles, syringes, broken glass- ware, and scalpels, may inadvertently puncture or scratch the skin and transmit the disease. Hand-to-mouth contact (with or without gloves) or contact with mucous membranes of the eyes and nose may cause infectious disease. There have been reported cases of HIV infections related to splashes of the eye with infectious agents.

A microorganism must, however, be viable, and the opportunity for infection must exist for an infection to occur. Temperature, humidity, and the presence or absence of growth factors affect viability. Mycobacterium tuberculosis is unaffected by drying and remains viable on work surfaces, whereas most other infectious agents cannot survive these conditions. Virulence varies among types and strains of microorganisms. Some microorganisms are highly pathogenic, even in healthy populations, but other microorganisms are opportunistic and are able to infect only hosts with reduced immunities. In fact, several medical conditions can increase the risk of infection. These include allergies, pregnancy, skin disorders, antibiotic therapy, and drugs that may alter host defenses.

The most likely mode of transmission concerning NMTs is direct person-person or person-patient contact. The second line of defense after PPE is hand washing frequently and after every patient contact situation, such as administering a dose to a patient or transferring a patient. Through adherence to Occupational Safety and Health Administration and Joint Commission on Accreditation of Healthcare Organizations standards, transmission of diseases can be minimized or eliminated.

\section{Biohazard Safety Classification}

There are 4 biosafety levels (BSLs) for working with infectious agents. The Centers for Disease Control, the National Institutes of Health, and the Nuclear Regulatory Commission have established recommended workplace guidelines for the containment of biohazardous agents at each level:

- BSL 1: work involving no known or minimal potential hazard;

- BSL 2: work with moderate-risk or indigenous agents associated with human disease;

- BSL 3: work with indigenous or exotic agents, with real potential for infection by aerosols and for the disease to have serious or lethal consequences;

- BSL 4: work with dangerous and exotic agents that pose a high individual risk of life-threatening disease or aerosol-transmitted laboratory infections or with related agents with an unknown risk of transmission.

In nuclear medicine laboratories, it would be rare to be involved in any level above BSL 2 (9).

\section{Low-Back Pain}

The major causes of low-back pain in technologists are continuous overuse of back muscles attributable to moving 
patients and moving patients in an improper manner. Two important concepts for proper lifting techniques are understanding the "base of support" when lifting and the "center of gravity" for both technologists and patients. The base of support is extremely important for maintaining balance during lifting. A typical example is the stance a football player takes once a play begins. Feet should be wide apart, with one foot in front of the other; this stance makes it difficult for an individual to be tipped over or, as in the example of the football player, to be tackled to the ground. The center of gravity is important for technologists as well as patients. In general, in an adult, the center of gravity is the midpoint of the pelvis at approximately $\mathrm{S}-2$ on the vertebral column. For infants and toddlers, the center of gravity is slightly higher and not over their base of support. This is why it is easy for toddlers to tip over and fall down when learning how to walk. It is also why it is easy for toddlers to fall into buckets and possibly drown.

When moving a patient, a technologist should keep the majority of the weight being moved as close to the technologist's own center of gravity as possible for a better mechanical advantage. This means close contact with a patient who is being moved. A technologist should always be aware of the patient's center of gravity and use the knowledge of where it is located to advantage, if possible, when moving a patient from a supine to a recumbent position or vice versa. Some principles for safe patient transfers include letting the patient do as much of the transfer as possible and within reason. A technologist should always establish a wide base of support for stability, should always lift with the legs, and should avoid bending or twisting of the back. Perhaps most important is that a technologist should never lift more than he or she can without injury and should always ask for assistance when needed; for example, a technologist who weighs $45 \mathrm{~kg}$ (100 lb) should ask for assistance with a patient who weighs $112.5 \mathrm{~kg}$ (250 lb) (10). It is wise to periodically have training on proper transfer techniques, perhaps biannually or when there is a large turnover of employees, to keep transfer techniques fresh in the minds of employees and to help reduce potential lowback problems.

\section{Potential Cumulative Trauma Disorders (CTDs) or Injuries}

CTDs or cumulative trauma injuries are not a diagnosis; rather, they are a group of disorders that have similar characteristics. Often referred to as repetitive trauma, these disorders are caused by repeated activities that cause the disorder to form. Several types of CTDs are possible in the clinical setting in nuclear medicine.

Myalgia is pain in one or more muscles, and tendonitis is inflammation of a tendon. Epicondylitis ("tennis elbow") involves inflammation of and pain in certain bony prominences in the area of the elbow, usually resulting from excessive strain on and twisting of the forearm. Trigger finger ("snapping finger") is a condition involving partial obstruction in flexion or extension of a finger; however, beyond the point of the obstruction, movement is eased. This condition is caused by constriction of a tendon sheath. Carpal tunnel syndrome is probably the most likely CTD that an NMT can develop. This disorder is caused by the compression of the median nerve in the carpal tunnel of the hand and is often associated with tingling, pain, or numbness in the thumb and first 3 fingers. The pain caused by carpal tunnel syndrome is enough to cause waking in the middle of the night. Raynaud's syndrome is probably the least likely CTD that an NMT can develop. It is an abnormal constriction of the blood vessels of the fingers on exposure to cold temperature and is usually caused by low-frequency-vibration hand tools. The destruction process occurs through contraction produced by pressure, which can reduce circulation. In addition, individual muscle fibers tear and joint surfaces rub together under pressure.

In addition to the repetitive motion of doing tasks the same way every day, day in and day out, there are risk factors associated with all CTDs. These risk factors include arthritis or other abnormal body and joint functions; cysts, swellings, and other abnormalities of tendons; poor circulation, which impairs repair mechanisms; fluid retention from circulation problems, high blood pressure, pregnancy, salt imbalance, or thyroid imbalance; poor dietary habits (e.g., lack of vitamin $\mathrm{B}_{6}$, which is important to the repair mechanisms of tissue); alcohol and tobacco use, which can affect the musculoskeletal system; nicotine, which is thought to break down connective tissue fibers; diabetes, which can affect circulation; previous injuries, which can affect and increase the likelihood of CTDs; and hormone shifts and even emotional stress, which can affect blood vessels by tightening them (11).

\section{CONCLUSION}

Occupational hazards for NMTs consist of a wide variety of possibilities, from the more obvious hazards of radiation and biologic safety issues to low-back pain and possible CTDs. It has been the intent of this article to briefly outline these possible hazards and to offer some information in a concise manner on how to avoid them in the clinical setting. Using time, distance, and appropriate shielding as well as standard or universal precautions will go a long way in reducing the more common safety concerns. Also, proper patient transfer techniques can help to reduce or eliminate low-back pain issues. CTDs are more difficult to prevent if an individual is doing the same activity during work day in and day out, but by knowing how to identify these disorders, an individual may be able to prevent them from becoming worse over time.

\section{REFERENCES}

1. Foshier S. Essentials of Radiation Biology and Protection. Albany, NY: Delmar Thomson Learning; 2002:60.

2. Dowd SB, Tilson ER. Practical Radiation Protection and Applied Radiobiology. 2nd ed. Philadelphia, PA: Saunders; 1999 
3. Lundberg TM, Gray PJ, Bartlett ML. Measuring and minimizing the radiation dose to nuclear medicine technologists. J Nucl Med Technol. 2002;30:25-30.

4. UCSF Office of Environmental Health and Safety. Radiation safety training manual, chapter 6: practical steps to radiation safety. Available at: http://www.ehs.ucsf. edu/Manuals/RSTM/RSTM\%20chap6a.htm. Accessed January 23, 2008.

5. Health Physics Society. Answer to question \#4716 submitted to "Ask the Experts." Category: medical and dental equipment/shielding-shielding. Available at: http://hps.org/publicinformation/ate/q4716.html. Accessed January 23, 2008.

6. Cherry SR, Sorenson JA, Phelps ME. Physics in Nuclear Medicine. 3rd ed. Philadelphia, PA: Saunders; 2003.
7. Saha GB. Physics and Radiobiology of Nuclear Medicine. 2nd ed. New York, NY: Springer-Verlag; 2001:204-205.

8. Centers for Disease Control and Prevention. Prenatal Radiation Exposure: A Fact Sheet for Physicians. Atlanta, GA: Centers for Disease Control and Prevention; 2005:1-6.

9. Wall DK. Industrial Hygiene Made Easy. Orange Park, FL: Moran Associates; 2000:129-141.

10. Adler AM, Carlton RC. Introduction to Radiologic Sciences and Patient Care. 4th ed. Philadelphia, PA: Saunders; 2007:163-177.

11. Brauer RL. Safety and Health for Engineers. New York, NY: Van Nostrand Reinhold; 1990:144-147. 